\title{
Violenza istituzionale contro le donne al tempo del Covid-19. Riflessioni pedagogiche
}

\author{
Institutional violence against women during the covid-19 epi- \\ demic. Pedagogical reflections \\ Silvia Leonelli*
}

\begin{abstract}
Riassunto
A partire dal febbraio 2020 il governo italiano ha iniziato a prendere iniziative per arginare la diffusione del virus Covid-19, che tuttavia non hanno tenuto conto del fenomeno della violenza domestica. L'invito a "restare a casa" è sembrato adatto a quasi tutta la cittadinanza, ma si è trasformato in una forma di maltrattamento aggiuntivo per tutte quelle donne che si sono trovate isolate $\mathrm{e}$, in pratica, segregate, assieme al loro persecutore. Altre disposizioni contenute nei decreti, poi, non hanno agevolato la loro ricerca di aiuto.

L'articolo si pone come primo obiettivo quello di analizzare un tipo particolare di violenza contro le donne, quella istituzionale, e di mostrare come essa sia scaturita dai vari decreti ministeriali che si sono succeduti durante l'epidemia, quali conseguenze abbia generato e come sia stato gestito questo problema in seguito, quando l'opinione pubblica si è mobilitata. Un secondo obiettivo è quello di inserire tale discorso all'interno degli studi pedagogici che si occupano del più ampio fenomeno degli abusi contro le donne.

Parole chiave: Violenza contro le donne. Violenza istituzionale. Pedagogia di genere.

Abstract

Since February 2020, the Italian Government has started to take initiatives to stem the spread of the Covid-19 virus, which have not taken into account the phenomenon of domestic violence (Intimate Partner Violence). The invitation to "stay at home" seemed suitable for almost all the citizens, but it has become a form of additional mistreatment for all those women who found themselves isolated and segregated, together with their persecutor. In addition, other provisions in the decrees did not facilitate their search for aid.

The first goal of the paper is to analyze a particular kind of violence against
\end{abstract}

* Ricercatrice in Pedagogia generale e sociale presso il Dipartimento di Scienze dell’Educazione “G. M. Bertin”, Università di Bologna. E-mail: s.leonelli@unibo.it.

Doi: 10.3280/ess1-2020oa9582 
women, the institutional one showing how it came out from the various ministerial decrees during the epidemic. We need to understand which consequences this problem generated and how it was consequently dealt, when public opinion mobilized. A second goal is to include this speech into the pedagogical studies dealing with the wider phenomenon of abuse against women.

Keywords: Violence against women. Institutional violence. Gender Pedagogy.

A Marianna Manduca

\section{Quando l'\#loRestoaCasa diventa pericoloso per le donne vittime di violenza: qualche dato preoccupante 1 .}

Nel febbraio 2020 lo Stato italiano ha iniziato a prendere iniziative per arginare la diffusione del nuovo Coronavirus sul territorio nazionale, e l'invito/obbligo di "restare in casa" è sembrato adatto a quasi tutta la cittadinanza, esclusi coloro che dovevano muoversi per ragioni di lavoro, salute o per necessità. Nelle prime settimane non ci si è resi conto che le misure di isolamento avrebbero avuto una grande ripercussione sulle donne vittime di violenza intrafamiliare (o domestica) ${ }^{2}$.

\footnotetext{
${ }^{1}$ Alcune precisazioni terminologiche. La definizione "vittima di violenza" viene utilizzata nonostante le perplessità espresse da numerose studiose, che sottolineano il rischio di inchiodare la donna a un'identità passiva, immutabile. Ci si avvale poi in via privilegiata della formula "violenza contro le donne", per delimitare il campo rispetto a "violenza di genere" che sarebbe più corretta ma che comprende anche, ad esempio, la violenza omofobica, non oggetto del presente contributo. "Abuso", "maltrattamento" e "violenza" sono adoperati come se fossero sinonimi, per agevolare la lettura evitando ripetizioni.

2 L'OMS nel 1996 ha affermato che la violenza nelle relazioni intime, Intimate Partner Violence, comprende abusi fisici, psicologici, sessuali, economici e persecutori (stalking) da parte di «soggetti che hanno, hanno avuto o si propongono di avere una relazione intima di coppia, quanto di soggetti che all'interno di un nucleo familiare più o meno allargato hanno relazioni di carattere parentale o affettivo» (World Health Organization, 1996). Qualche anno dopo la stessa organizzazione ha precisato che: «La violenza familiare e contro i partner intimi avviene tra i membri della famiglia e i partner intimi, e di solito, anche se non esclusivamente, si svolge in casa. Essa si verifica in tutti paesi, tutte le culture e a ogni livello della società senza eccezioni (World Health Organization, 2002)». Le espressioni” violenza domestica", "v. familiare" e "v. intrafamiliare" vengono qui usate in modo interscambiabile.
} 
Va ammesso che la drammatica situazione in cui versava il nostro paese nei mesi di febbraio e marzo aveva fatto accantonare l'attenzione nei confronti di alcuni problemi sociali che poi puntualmente, in seguito, sono esplosi.

Quando le norme nazionali e regionali si sono fatte progressivamente più restrittive, e quando si è colto che il lockdown domiciliare sarebbe durato a lungo, i Centri Antiviolenza e le associazioni di donne hanno espresso numerose apprensioni. Forti di un'esperienza consolidata (gli accessi aumentano al termine delle vacanze estive e di quelle invernali), hanno ricordato a tutti che la convivenza forzata tra coniugi sotto lo stesso tetto in taluni casi può rivelarsi pericolosa. Se la violenza è già presente nella vita della coppia, può farsi più frequente, o peggiore; se emerge ex-novo può essere innescata anche dalla coesistenza obbligata della coppia, e dalla frustrazione del periodo. Occorre però precisare l'ultima frase: non va dimenticato che, nella maggior parte dei casi, a monte del maltrattamento c'è un comportamento strategico tenuto dall'uomo, ovvero c'è un percorso che viene compiuto. È scorretto dire che gli abusi sono stati causati dal \#IoRestoaCasa, perché equivale a ricadere nell'obsoleto immaginario per il quale c'è un raptus che scatta all'improvviso, come dire: un fulmine a ciel sereno. Non è così.

Una parte del problema è certamente rappresentata dalla segregazione della coppia, l'altra dalla solitudine delle vittime. Chiuse in casa, lontane dalle loro figure di riferimento, private della possibilità di movimento autonomo, $\mathrm{o}$ potendo fare spostamenti limitati e controllati, impaurite dall'idea di telefonare ai Centri Antiviolenza mentre il marito/compagno è nella stanza accanto e può sentirle, alcune tra loro si sono trovate davvero sole.

Così si è verificata una circostanza allarmante: da un lato, il probabile aumento della violenza in famiglia, con il lockdown a fare da detonatore o da amplificatore, dall'altro l'impossibilità per le donne di ricorrere a quei servizi in grado di aiutarle. Vediamo qualche dato.

A livello nazionale, il numero telefonico 1522, promosso dalla Presidenza del Consiglio dei Ministri e dal Dipartimento per le Pari Opportunità, ha registrato un rilevante calo di chiamate. Le volontarie del Telefono Rosa, che gestiscono la linea telefonica, hanno confrontato le segnalazioni dell'anno 2019 e dell'anno 2020, notando che:

Lo scorso anno nelle prime due settimane di marzo le donne vittime di violenza o le segnalazioni di casi, erano state 193 (164 italiane pari all' $85 \%$ e 29 straniere pari al $15 \%$ ). Quest'anno, invece durante l'emergenza, nelle prime due settimane di marzo le vittime di violenza (o le segnalazioni di violenza) che hanno chiamato il telefono dedicato sono state 101 (1'86\% italiane e il $14 \%$ straniere) con una diminuzione del $47,7 \%$. Sono praticamente crollate le telefonate di vittime di stalking: l'anno scorso nelle prime due settimane di marzo erano state 33 (91\% italiane e 9\% straniere), que- 
st'anno soltanto 7 (sei italiane e una straniera) segnando una diminuzione del 78,8\% (Mind the gap, 2020).

Il servizio 1522 è solo uno tra dei "nodi della rete" che può assistere le donne, ma sul territorio ci sono altre possibilità: Centri antiviolenza, associazioni, pronto soccorso, assistenti sociali, forze dell'ordine, consultori familiari, ecc. Tutti hanno segnalato il medesimo inciampo, il calo dei contatti, che viene riportato, ad esempio, nei siti dei Centri, e in quelli di D.i.Re-Donne in Rete contro la violenza, ma senza supporto di dati ${ }^{3}$.

Ne abbiamo invece per la zona di Milano e per la Lombardia, che è stata la prima Regione nella quale il virus ha manifestato con forza la propria pericolosità e che purtroppo -ma speriamo di no -può essere considerata un caso emblematico di ciò che potrebbe accadere in tutto il territorio nazionale. Sono dati interessanti perché provengono da tre attori diversi: un ospedale, un'associazione e la Procura di Milano (Chiale, 2020).

Dal lato ospedale, il servizio Svsed-Soccorso violenza sessuale e domestica del Policlinico di Milano segnala che l'anno scorso, dal 24 febbraio al 25 marzo 2019, c'erano stati 73 nuovi accessi, mentre quest'anno si sono ridotti a 43. Dal lato associazione, la Fondazione Somaschi, che peraltro gestisce alcuni Centri antiviolenza, ha evidenziato un calo del $69 \%$ nelle chiamate telefoniche al numero dedicato. La Procura di Milano, infine, non fornisce numeri o paragoni con lo scorso anno, ma testimonia che sono calate le denunce per maltrattamenti e mette in luce che, in aggiunta, «con le misure in atto, vengono meno i sistemi di controllo informali su possibili violenze come la scuola, il bar, la chiesa, oltre al ruolo degli assistenti sociali». La responsabile di area della Fondazione Somaschi ha poi constatato che sono raddoppiate le richieste che arrivano dalle forze dell'ordine: se i servizi "intermedi" sono irraggiungibili per una serie di ragioni, la donna che si trova in difficoltà scappa in caserma, oppure sono i carabinieri e la polizia ad accorrere durante/dopo un episodio violento. La donna cioè chiede aiuto solo quando il maltrattamento giunge all'acme, e questa gestione in emergenza, talvolta, può far degenerare la situazione, in particolare se la donna deve poi tornare al proprio domicilio. Quando la violenza raggiunge l'apice la vittima corre rischi più gravi. Invece, la responsabile del servizio Svsed sopracitato aggiunge una sfumatura diversa a proposito del calo dei contatti, che riguarda in un certo senso la violenza psicologica. Un tratto abbastanza comune nei maltrattanti è l'atteggiamento di controllo della donna, che perde la sua autonomia. In questo momento di chiusura in casa insieme, il maltrattante ovviamente può esercitare il controllo

\footnotetext{
${ }^{3}$ La rete D.i.Re raggruppa 80 organizzazioni che si occupano della violenza di genere; esse gestiscono numerosi sportelli e numeri verdi, e circa un terzo di tutti i Centri antiviolenza italiani (https://www.direcontrolaviolenza.it/).
} 
in maniera totale e questo «può rendere l'escalation di violenza meno probabile». In questa ipotesi, i maltrattamenti ci sono ma non raggiungono il culmine; la donna li vive quotidianamente e tuttavia, data l'epidemia di Covid-19, aspetta a chiedere sostegno, quando invece in tempi normali sarebbe già andata a denunciare (tutti i dati di questi tre enti sono tratti da: Chiale, 2020).

Sempre dalla Lombardia arrivano ulteriori cifre che confermano la contrazione dei contatti. Lo Sportello Donna-Centro antiviolenza di Treviglio ribadisce il calo dei nuovi accessi: se la media nel 2020 è stata di un caso al giorno, da inizio marzo è stata di un caso ogni quattro giorni; l'associazione Aiuto Donna di Bergamo, poi, comunica che il numero di telefonate ricevute è «quasi a zero» (Offredi, 2020).

Per comprendere la portata di questi dati, bisogna considerare che il sommerso era già elevatissimo prima del Covid-19. Le violenze non solo non venivano denunciate, ma non venivano neppure raccontate. Più o meno una donna su quattro, tra le vittime interpellate dall'Istat, non ne parlava con nessuno:

La violenza di genere è un fenomeno ancora sommerso, è elevata, infatti, la quota di donne che non parlano con nessuno della violenza subita (il $28,1 \%$ nel caso di violenze da partner, il $25,5 \%$ per quelle da non partner), di chi non denuncia (i tassi di denuncia riguardano il $12,2 \%$ delle violenza da partner e il $6 \%$ di quelle da non partner), di chi non cerca aiuto; ancora poche sono, infatti, le donne che si rivolgono ad un centro antiviolenza o in generale a un servizio specializzato (rispettivamente il $3,7 \%$ nel caso di violenza nella coppia e l'1\% per quelle al di fuori). Ma la cosa più preoccupante è che queste azioni sarebbero davvero essenziali per aiutare la donna ad uscire dalla violenza (Istat, 2014).

La situazione è fosca: già la violenza non veniva detta quando c'era la possibilità, adesso poi è diminuita anche quella piccola quota di segnalazioni e di richieste di sostegno. Per usare le parole dei media, i maltrattamenti restano ancora di più nascosti dentro le mura domestiche: al tempo del Covid-19 la casa, quello spazio (mentale, emotivo, non solo fisico) che dovrebbe essere improntato al benessere, può diventare per alcune una prigione soffocante.

Si tratta di una preoccupazione che non riguarda solo il nostro paese, tanto che il 27 marzo 2020 il Consiglio per i diritti umani delle Nazioni Unite, nella persona di Dubravka Simonovic, relatrice speciale per la violenza contro le donne, ha lanciato un allarme globale. Simonovic ha invitato gli Stati membri ad attivarsi, dal momento che:

È altamente probabile che il livello della già diffusa violenza domestica aumenti, come già suggerito da indicazioni preliminari di polizia e operatori. Per fin troppe donne e bambini la casa può essere un luogo di paura e abuso. Una situazione che si 
aggrava considerabilmente in casi di isolamento come il lockdown imposto nell'emergenza Covid-19». (...). A peggiorare le cose, restrizioni di movimento, vincoli finanziari e incertezza generalizzata incoraggiano i maltrattanti e forniscono loro ulteriore potere e controllo (Onu, 2020).

Ritornando ai dati italiani a disposizione, bisogna chiarire che quando le donne chiamano il 1522 oppure contattano un Centro antiviolenza, o un qualsiasi ente della rete, non stanno facendo denuncia. Quindi è possibile che, al termine dell'epidemia, si possa riscontrare una discrepanza tra i numeri, cioè tra i pochi contatti alle associazioni (agli sportelli, ai servizi ospedalieri e sociosanitari, ecc.), e l'aumento del numero di denunce presso le forze dell'ordine. Però, come si diceva sopra, l'ipotesi è che possano crescere le segnalazioni dei reati più gravi, quelli efferati, che hanno fatto pensare alle vittime di rischiare la loro incolumità e quella dei figli.

Perimetrare la "vera" dimensione della violenza intrafamiliare in Italia, come si comprende, è molto difficile perché si tratta di incrociare informazioni provenienti da molti soggetti (istituzionali, associativi, ricerche accademiche, rilevazioni periodiche dell'Istat, ecc.) che hanno mission diverse tra loro. Solo a posteriori sapremo se gli abusi sono aumentati per via dell'isolamento domiciliare, come si teme.

Il fenomeno della violenza, però, era già ragguardevole prima. L'Istat (2014) ha stimato in 496mila le donne che hanno subito una qualsiasi forma di violenza nelle relazioni di coppia, nei 5 anni precedenti il 2014. La violenza non proveniva da un ex ma proprio dall'uomo al quale erano ancora legate; peraltro, partner o ex partner erano quelli che commettevano le violenze più gravi. Inoltre, tra quelle che avevano subito abusi da un partner o un ex partner, come visto sopra, solo il 3,7\% si era rivolta a un centro antiviolenza o a un servizio per un sostegno (nel 2006, anno della precedente rilevazione, erano state il 2,8\%). Tirando le somme: in cinque anni, circa mezzo milione di vittime hanno formulato pochissime richieste di aiuto e/o denunce.

A proposito di denunce: la Polizia di Stato segnala che nel 2019 ogni giorno dell'anno 88 donne hanno subito maltrattamenti, stalking, violenza sessuale e percosse (Polizia di Stato, 2020, a). Nello stesso Rapporto si legge che: «Nell' $82 \%$ dei casi chi fa violenza su una donna ha le chiavi di casa». C'è poi un dato utile per una comparazione: nel mese di marzo 2019 ogni 15 minuti circa è stata registrata una vittima di violenza di genere di sesso femminile (non solo in ambito familiare). Possibile che casualmente, un anno dopo, $\mathrm{i}$ maltrattamenti domestici si siano quasi azzerati durante l'epidemia del Covid19 ? 


\section{Del perché il sapere pedagogico debba interessarsi della violenza istituzionale}

Il fenomeno della violenza contro le donne ha iniziato a essere indagato da parte del sapere pedagogico dopo essere stato oggetto di analisi in ambito sociologico, antropologico, psicologico, giuridico, ecc. È occorso un passaggio significativo, realizzatosi quando gli studi hanno dimostrato che gli abusi non possono essere attribuiti a una presunta aggressività biologica del maltrattante (causata dall'ormone del testosterone); quando si è colto che la violenza non può essere allontanata dal nostro sguardo semplicemente derubricandola al singolo maltrattante (attribuendola di volta in volta all'abuso di alcool-droga, a problemi psichiatrici, al disagio economico, allo scarso patrimonio culturale, alla classe sociale svantaggiata, ecc.); inoltre quando si è inteso che, nella sua versione domestica, la violenza non va considerata come un fatto privato, riguardante una coppia specifica, e va analizzata dal punto di vista pedagogico, anche per le conseguenze sugli eventuali figli e figlie.

Queste tre considerazioni ostacolavano la possibilità che i saperi dell'educazione potessero esprimersi compiutamente, che trovassero uno spazio di manovra. La prima faceva passare il messaggio che la violenza fosse un "destino biologico" sul quale l'intervento educativo avrebbe avuto scarsi o nulli risultati; la seconda e la terza convinzione davano l'idea che la violenza fosse un atteggiamento/comportamento imprevedibile, di un singolo uomo "sfortunato" o di una singola coppia "sfortunata", e che non fosse possibile organizzare percorsi e interventi di prevenzione pedagogicamente fondati.

Nel 2016 è stato pubblicato un Dossier pedagogico centrato sul tema della violenza (non solo quella rivolta contro le donne), dove si trova una frase che ha il pregio di descrivere bene come la patologizzazione del singolo caso, in passato, abbia frenato il discorso: «Se finora ci si è rifugiati in affermazioni quali: "Ma questo riguarda pochi casi patologici!", ora non ci si può più nascondere in una siffatta nicchia difensiva, perché la non volontà di vedere il disagio lo ha, di fatto, alimentato» (Corsi, Fabbri e Riva, 2016 b, p. 9).

La progettualità che caratterizza la pedagogia, rivolta al cambiamento culturale e politico (in senso alto), ha potuto dispiegarsi quando le ricerche si sono fatte più complesse. Come afferma l'Organizzazione mondiale della Sanità, riprendendo U. Bronfenbrenner (1979), la violenza è un fenomeno che origina al crocevia di molti fattori (biologici, sociali, culturali, economici e politici) senza che si possa identificare una causa/effetto lineare; per tentare di eliminarla, dunque, bisogna applicare modelli interpretativi di tipo ecologico (WHO, 2002). Lo sguardo deve spingersi fino ad arrivare a cogliere sia le molteplici forme di violenza sia ciò che non sembra esercitare un influsso sulla vita dei singoli, mentre invece sta interagendo con altri livelli di esperienza, in modo inavvertito. Difatti, il sapere pedagogico che esplora la violenza di 
genere ha già affrontato, e porta avanti da decenni, indagini su molti fronti. Alcune possono sembrare, come dire, più centrate e di maggiore spendibilità "diretta" nei contesti educativi, quali ad esempio: il femminicidio; la violenza assistita; quella di stampo omofobico; le infinite forme di abusi contro il corpo delle donne, ecc. (Ulivieri, 2014). Altre possono apparire più lontane dal piano della "gestione pratica" del problema, tuttavia sono altrettanto importanti per delineare un quadro approfondito: i modelli educativi violenti nella storia delle bambine; la violenza consentita dalle tecnologie della riproduzione; quella simbolica; i condizionamenti culturali in grado di riprodurre asimmetrie tra i generi; gli stereotipi sessisti veicolati dai media e quelli contenuti nei prodotti culturali rivolti alle giovani generazioni, ecc. (Corsi e Riva, 2013 a; Ulivieri, 2014). Si tratta comunque di approcci centrati su quegli aspetti socio-culturali che collocano il femminile e il maschile in posizioni dicotomiche e complementari, tali da aumentare la disparità tra i sessi. Inoltre, tali studi sondano le relazioni di potere tra i singoli, quelle che discendono da paradigmi culturali in grado di minare le pari opportunità.

Anche la violenza istituzionale, oggetto del presente contributo, può sembrare distante: in fin dei conti, quando lo Stato è oppressivo nei confronti delle donne, noi pedagogisti/e come possiamo agire? Quali percorsi formativi possiamo ideare per scardinare qualcosa che proviene dallo Stato? Che cosa possiamo proporre a scuola e nei contesti educativi, in grado di modificare la situazione? La violenza istituzionale, come si vedrà nei par. 3 e 4 , è un problema collettivo, e si verifica quando le istituzioni «non si impegnano a fondo nell'applicare la legge vigente» (non puniscono il maltrattante, non attivano finanziamenti per le iniziative di sensibilizzazione...). Inoltre, essa si palesa quando le istituzioni, in generale, non riescono a «garantire la sicurezza delle cittadine», e quando, in particolare, non si adoperano per «mettere in sicurezza le vittime» (Cretella e Romero, 2017, pp. 22 e 42). Il tutto pare fuori dalla portata dal nostro raggio di azione. Eppure, il modello ecologico, e se vogliamo il paradigma della complessità (Morin, 2001), esorta a portare gli studi in ogni direzione, perché ciascun taglio di analisi può illuminare aree nascoste del tema-violenza, non meno perniciose delle altre. Secondo tale approccio, i tentativi di risoluzione vanno ugualmente condotti su più fronti: «Oltre a contribuire a chiarire le cause della violenza e le loro complesse interazioni, il modello ecologico suggerisce anche che, al fine di prevenire la violenza, è necessario agire in diversi livelli allo stesso tempo» (WHO, 2002).

Seguendo dunque la teoria dello sviluppo umano (o bioecologico, Bronfenbrenner, 1979 e 2005) si può dire che la violenza istituzionale si alimenta nel macrosistema, ovvero in quel livello composto dai valori che permeano una società, dall'ideologia, dalla legislazione, dalle strutture politicoamministrative, dalle credenze e abitudini della propria cultura, e dei propri 
gruppi di riferimento. Però, se pure la violenza istituzionale si colloca al livello più remoto, le interconnessioni la rendono avvertibile in ogni contesto, dal momento che ci sono: «tanti cerchi concentrici, e ciascuno rimanda e si connette all'altro, ciascuno trae spessore e significato da ciascun cerchio che, a sua volta, concorre a definire» (Contini, 2002, p. 12). Ciò che accade nell'hic et nunc del quotidiano può essere condizionato dal «contesto che non c'è», da ciò che è poco visibile perché resta inavvertito sullo sfondo (Ivi).

In questo senso, gli studi sulla violenza istituzionale contro le donne, svolti in ambito pedagogico, costituiscono un affondo, un modo per esplorare qualcosa che, seppur lontano, è comunque presente e può far inceppare le nostre attività. Quando il mondo della formazione si occupa di violenza già analizza la cornice socio-culturale, dunque si tratta solo di aggiungere al discorso questo ulteriore aspetto, legato allo Stato. Infatti, in ambito educativo viene sempre compiuta una costante ricognizione del più ampio orizzonte in cui cresciamo e viviamo, per verificare che esso non porti avanti contenuti, rappresentazioni, valori volti a rendere inferiore il femminile (come categoria socioculturale) e tutte le altre differenze. Permeato dalla storica asimmetria di potere tra i sessi, lo zeitgeist contemporaneo resta pervaso da un ordine di genere molto stereotipato, che rende di fatto possibile l'accettazione (seppur dissimulata) della violenza e la sua legittimazione (seppur inconsapevole) in tutti i "cerchi concentrici". Il campo di analisi pedagogica, dunque, deve includere l'ordine simbolico, dal momento che: «le radici del male non vanno cercate nelle "anomalie" delle singole storie, delle infanzie ferite, dei raptus imprevedibili, ma nella normalità di un ordine simbolico intrinsecamente violento» (Musi, 2014, p. 53). Certo, la violenza nasce dalla volontà di un singolo, che non va in alcun modo deresponsabilizzato. Non ci sono alibi. Inoltre, non si può negare che i cerchi più vicini all'individuo esercitino un peso notevolissimo e vadano ricompresi nelle nostre analisi: «le norme giuridiche sono un quadro all'interno del quale sono la società, la famiglia, la scuola che devono mutare atteggiamento» (Ulivieri, 2013 b, p. 174). Abitualmente, gli approfondimenti sulle coordinate culturali vanno ad affiancare il lavoro svolto con quei progetti che da una trentina d'anni si occupano di educazione alle emozioni, educazione al conflitto, educazione sentimentale, educazione di genere, ecc. Essi sono da intendersi, anche, come tentativi di far aumentare la consapevolezza alle giovani generazioni su quanto c'è di costruito socialmente nel rapporto tra $\mathrm{i}$ sessi. Dunque, su ciò che è modificabile laddove si riscontrino discriminazioni e atteggiamenti/comportamenti non rispettosi delle differenze.

Però, a causa dell'epidemia di Covid-19, la parte formativa sul campo (cioè, ad esempio, i laboratori nei quali si lavora con gli/le adolescenti) si è dovuta arrestare. Così pure le ricerche in presenza. In questo momento in Italia le scuole e le agenzie educative sono chiuse: ciò che si può fare contro la 
violenza sulle donne è come sospeso. Ma le indagini sul tema vanno avanti; in questo periodo così particolare può essere sviluppata maggiormente la matrice teorica della pedagogia, per realizzare le sue due istanze principali, quella critica e quella regolativa (Cambi, 2009). Detto in altri termini, a proposito della violenza:

occorre senz'altro una pars destruens, di critica radicale e coraggiosa di costumi, modelli, abitudini, connivenze, quieto vivere, seguita da una pars construens, che escogiti modalità inedite (questa volta efficaci, perché fondate su un'analisi profonda degli aspetti solitamente tenuti nascosti o negati) di co-costruzione della salute e del ben-essere personale e collettivo (Corsi e Riva, 2013 b).

Se pure in questa fase non è possibile agire nei contesti educativi, è fattibile prepararsi a ciò che verrà, e svolgere il lavoro di decostruire i condizionamenti e costruire le premesse per una diversa concezione del femminile e del maschile (socialmente intesi), proprio perché «alla pedagogia, più che a ogni altra disciplina, spetta il compito di individuare pratiche e dispositivi inediti con cui riformulare l'ordine simbolico che incornicia le relazioni tra i soggetti» (Musi, 2014, p. 55).

\section{La violenza istituzionale contro le donne}

La violenza istituzionale intreccia dimensioni politiche, economiche, legislative, sociali e culturali. Si trova espressa nei provvedimenti, nelle sentenze dei tribunali, nelle leggi, nelle pieghe di una burocrazia che ostacola singole persone o gruppi di soggetti nell'esercizio dei loro diritti di pari opportunità (goduti invece dal resto della comunità). Essa non agevola l'uscita da specifiche situazioni di difficoltà e discriminazione: «La violenza istituzionale è quella permessa dallo Stato ed è presente nelle istituzioni e nell'amministrazione pubblica. Essa non permette alle donne di accedere ai propri diritti fondamentali e/o ne blocca il godimento reale ed effettivo» (Cretella e Sanchez, 2014, p. 73).

Già nel 1993 la Dichiarazione sull'eliminazione della violenza contro le donne (Onu) riconosceva il problema, affermando che è da intendersi come violenza anche quella: «fisica, sessuale e psicologica perpetrata o condotta dallo Stato, ovunque essa accada» (art. 2, comma c). Inoltre, l'Onu chiedeva agli Stati di: «Esercitare la dovuta attenzione per prevenire, indagare e, conformemente alla legislazione nazionale, punire gli atti di violenza contro le donne, sia che tali atti siano perpetrati dallo Stato che da persone private» (art. 4, comma c). 
Tuttavia, dopo quasi trent'anni si può convenire che la violenza istituzionale è ancora poco conosciuta nel senso comune rispetto alle altre forme di abusi, nonostante sia stata l'oggetto di studi e riflessioni accademiche e/o del sapere esperto. Negli ultimi anni si è iniziata a diffondere una certa cultura rispetto alle caratteristiche dello stalking, della violenza psicologica, di quella economica, di quella domestica-intrafamiliare, e, ancora non compiutamente, si è iniziato a parlare di quella assistita, vissuta dalle figlie e dai figli che assistono a episodi di maltrattamento, o che li subiscono. I temi inerenti ai maltrattamenti fisici e sessuali, essendo più intuitivi, erano già presenti allo sguardo della popolazione da alcuni decenni, anche se, ancora oggi, restano zavorrati da numerosi stereotipi. Tutte queste configurazioni della violenza, comunque, sono molto più padroneggiate dal dibattito pubblico rispetto a quella istituzionale. Va segnalato che essa può riguardare gli uomini, ad esempio a causa del loro orientamento sessuale, ma tendenzialmente colpisce le donne perché comunque l'ordine simbolico di genere che caratterizza il mondo occidentale è ancora connotato da elementi sessisti:

L'origine di squilibri e iniquità tra i generi si radica molto prima della violenza $\mathrm{e}$ delle sue zone rosse di allerta e di pericolo, di scandalo ed eccentricità, interroga l'educazione non solo in relazione a oggetti di studio ben definiti (i saperi, le rappresentazioni mediatiche, le politiche sociali...) ma nella sua responsabilità di applicarsi all'analisi dei processi che li determinano, delle logiche sottese alla costruzione dell'ordine simbolico (...) (Musi, 2014, p. 54, corsivo mio).

La teorizzazione del concetto di violenza istituzionale ha preso vigore grazie all'esame dei fatti di Ciudad Juerez, in Messico, dove i soprusi e l'omicidio delle donne negli anni Novanta avevano raggiunto tali proporzioni da imporre una riflessione internazionale. Marcela Lagarde, e prima ancora Diana Russell, si erano interrogate sui termini di femicidio e femminicidio, fino a identificare quest'ultimo come un vero e proprio "crimine di Stato" (Spinelli, 2008). Lo Stato può essere indifferente/silente/trascurante oppure ostile:

La violenza istituzionale si manifesta nell'ostilità verso la categoria del femminile, nell'indifferenza dello Stato e nella sua responsabilità nel sottacere il fenomeno, nel trascurare adeguate azioni di prevenzione, quali diffuse campagne di coscientizzazione volte a riconoscere e contrastare le violenze legate a pregiudizi e stereotipi (Pinto Minerva, 2013, a, p. 162).

Tale avversione può manifestarsi in modo dissimulato (ad esempio promettendo fondi per contrastare la violenza che poi non vengono erogati, o rendendo le procedure talmente cavillose da renderli inaccessibili) oppure può 
essere agita. Infatti, traspare una sorta di intenzionalità in una serie di condotte messe in opera dalle istituzioni, spesso una vera:

responsabilità delle istituzioni nel non aver saputo mettere in atto iniziative di prevenzione alla violenza, di avere sottovalutato le molteplici forme di annientamento dell'identità femminile attraverso procedure di assoggettamento, soprusi e sottomissione, fino alla morte delle vittime (Pinto Minerva, 2013, a, p. 162).

Va segnalata una forte assonanza tra i concetti di violenza istituzionale e di violenza strutturale, ma quest'ultimo concetto è più esteso e si riferisce a: «un'organizzazione sociale che permette la violenza contro le donne e la giustifica attraverso tradizioni e credenze culturali, religiose, ideologiche, politiche e sociali» (Cretella e Sanchez, 2014, p. 72). Si tratta di un sistema che agisce in modo diretto e indiretto, e che, come ci spiegano gli studi antropologici, «ha a che fare con i rapporti di potere tra uomini e donne nelle diverse società» (Ibidem). Riprendendo P. Farmer, è possibile dire che quando un' organizzazione non affronta, e non risolve, le disuguaglianze tra i suoi membri, e quando crea/accentua le differenze di potere, e di accesso alle risorse fondamentali, diviene essa stessa violenta (Quaranta, 2006).

Ritornando alla violenza istituzionale, ancora manca una consapevolezza diffusa del fatto che lo Stato (nelle sue varie declinazioni) possa risultare violento, e che alcuni organismi pubblici possano ostacolare le donne, anziché aiutarle come invece essi affermano esplicitamente.

In qualche caso, anche la rete di sostegno alle donne, della quale si è parlato sopra, mostra i segni di una «complicità pubblica» alla violenza (Romito, 1999 e 2000). Infatti, la collusione con un ordine di genere discriminatorio può essere portata avanti da persone che, nel loro ruolo, incarnano lo Stato: operatori socio-sanitari, personale dei consultori e dei servizi territoriali d'accoglienza, forze dell'ordine, forze di giustizia, ecc ${ }^{4}$. Tra l'altro, va ag-

\footnotetext{
${ }^{4}$ La violenza istituzionale contro le donne si può constatare in molte altre sfere, che qui non possono essere analizzate per esigenze di sintesi: giusto per fare un esempio, nel fenomeno denominato "vittimizzazione secondaria", riscontrabile durante l'iter legale (denuncia, processo...) e sanitario che si percorre quando si tenta di uscire dalla situazione. Ma gli ambiti nei quali agisce la violenza istituzionale sono numerosi: dal mondo del lavoro a quando lo Stato legifera a proposito della maternità, della contraccezione, ecc., e/o quando organizza servizi centrati sulla salute delle donne che però, spesso, si trasformano in luoghi nei quali esse vengono in realtà espropriate dell'esperienza del loro corpo (Galelli, 2014). Si può parlare di un vero e proprio esercizio di potere, operato dallo Stato, volto a disciplinare i corpi femminili e la sessualità femminile, laddove si intenda il corpo come una «realtà biopolitica» e non come soggettività: «Con il termine "biopolitica" mi riferisco a quello che Foucoult ci insegna essere l'insieme delle pratiche, dei discorsi e dei dispositivi posti in essere da istituzioni politiche e sociali, da centri di potere, da centri di ricerca, di produzione e di utilizzazione di saperi e conoscenze, volti alla normazione e al controllo dei soggetti. Più in particolare, volti alla normazio-
} 
giunto che tale assetto simbolico è talmente pervasivo da impregnare talvolta le stesse strategie istituzionali di contrasto alla violenza:

La violenza contro le donne dimostra dunque radici talmente profonde nella nostra cultura - dalle forme di organizzazione della nostra società al nostro immaginario, dal nostro linguaggio alle nostre rappresentazioni dei ruoli sessuali - che anche le strategie istituzionali di contrasto, le nostre reazioni indignate e le nostre domande di punizione rivelano di condividere inconsapevolmente lo stesso universo simbolico, lo stesso substrato culturale, in cui quella violenza trova le sue origini e si sviluppa e in cui si genera quell'oppressione, quella miseria delle relazioni e delle emozioni (Ciccone, 2009, p. 21, corsivo mio).

In altri casi, è proprio il complesso delle leggi ad aggiungere violenza a violenza. La vicenda di Marianna Manduca, al proposito, è emblematica (De Luca, 2020). Siamo nel 2007, Marianna ha sporto ormai 12 denunce contro il marito per violenza e minaccia a mano armata senza ricevere protezione (forse neppure ascolto) da parte di quell'apparato statale che doveva tutelarla. Viene uccisa, e i suoi tre figli minorenni vengono affidati a un cugino, e poi da lui adottati. Lo Stato - ovvero la Corte d'appello di Messina - in primo grado, al termine del processo, riconosce la responsabilità civile dei magistrati che non sono intervenuti durante tutto il periodo precedente l'omicidio al fine di scongiurarlo. I figli ricevono, a titolo di risarcimento, 250.000 euro. Nel 2019 la Corte di appello, in secondo grado, annulla il risarcimento, perché la Presidenza del Consiglio ha presentato ricorso, e dunque pretende che i figli restituiscano la somma. La motivazione addotta è che l'omicidio fosse «invitabile», si sarebbe compiuto ugualmente, dato che il marito era determinato a farlo. Una doppia violenza istituzionale, a ben guardare: da un lato, uno Stato che, in primo grado, ammette che i suoi magistrati hanno commesso omissioni; dall'altro uno Stato che, in secondo grado, afferma di non poter aiutare una donna vittima di maltrattamenti se il marito intende davvero ucciderla. Al momento, i figli hanno rivolto un appello al Presidente della Repubblica, Sergio Mattarella, e attendono l'esito del ricorso in Cassazione.

Eppure, si tratta dello stesso Stato che già l'anno prima, nella legge 11 gennaio 2018, n. 4, «Modifiche al codice civile, al codice penale, al codice di procedura penale e altre disposizioni in favore degli orfani per crimini domestici», all'articolo 8 normava l'accesso ai servizi di assistenza agli orfani per crimini domestici (ma senza nuovi o maggiori oneri per la finanza pubblica). Ed è lo stesso Stato che nella legge 19 luglio 2019, n. 69, «Modifiche al codice penale, al codice di procedura penale e altre disposizioni in materia di tute-

ne e al controllo che passa attraverso gli aspetti più minuti e, per questo, anche più radicali della vita di ciascuno, ossia attraverso il corpo» (Galelli, 2014, p. 131). 
la delle vittime di violenza domestica e di genere», all'articolo 8, prospetta diverse forme di sostegno economico in favore degli orfani per crimini domestici (e delle famiglie affidatarie).

Nel nostro paese esistono leggi che sanzionano pesantemente chi commette i reati di stalking e revenge porn, gli autori di violenza fisica, psicologica, ecc., possibile che lo Stato sia, da un lato il sanzionatore, e dall'altro il maltrattante?

\section{La violenza istituzionale contro le donne vittime di violenza, nelle Circolari an- ti Covid-19}

L'epidemia di Coronavirus in Italia permette di percepire come possano manifestarsi alcune forme di violenza istituzionale. I vari decreti del Presidente del Consiglio, del Ministero dell'Interno e del Ministero della Salute, che si sono succeduti a partire dal mese di febbraio 2020 (e le varie disposizioni regionali, non sempre in linea con quelle nazionali) hanno aggiunto difficoltà alle vittime e a chi, come i Centri Antiviolenza, le affianca e le sostiene durante il percorso ${ }^{5}$. La confusione tra le norme, che si sono andate anche sovrapponendo, e i cambiamenti che esse hanno generato, hanno creato situazioni critiche.

Immaginiamo una donna che ha iniziato a subire violenza da quando lei e il coniuge/compagno sono rimasti isolati nella casa familiare, e ha capito di volervi porre rimedio. Oppure, immaginiamone una che stava già affrontando l'iter di uscita dalla violenza. Poniamo che la donna viva in un paese di provincia dove non c'è un Centro Antiviolenza ${ }^{6}$ e si debba muovere in auto per raggiungerlo, oppure che voglia rivolgersi a uno sportello di sostegno psicologico lontano da casa, o chiedere un consiglio legale, o prendere contatto con i servizi sociali, ecc. Che cosa può scrivere nell'autocertificazione che serve a giustificare gli spostamenti? Questo è un tassello di violenza istituzionale:

${ }^{5}$ Nel 2019 l'Istat ha pubblicato la prima indagine sui 281 Centri antiviolenza italiani (CAV), a seguito di una ricognizione del 2017. In quell'anno 43.467 donne si erano rivolte ai CAV ma solo il 67,2\% aveva iniziato un percorso di uscita dalla violenza. Il Rapporto dettaglia la popolazione di riferimento: «tra le donne che hanno iniziato tale percorso, il $63,7 \%$ ha figli, minorenni nel $72,8 \%$ dei casi. Le donne straniere costituiscono il $27 \%$ di quelle prese in carico» (Istat, 2019).

${ }^{6}$ L'art. 4 dell'Intesa Stato Regioni del 2014 stabilisce ciò che i Centri antiviolenza devono garantire: ascolto, accoglienza (telefonica o mediante colloqui), assistenza psicologica, legale, creazione di gruppi di auto-aiuto, ecc. Alcuni CAV riescono a offrire anche servizi di supporto ai minori vittime di violenza o violenza assistita, orientamento al lavoro, aiuto burocratico e amministrativo, orientamento all'autonomia abitativa $e$ ospitalità in casa rifugio. (https://www.gazzettaufficiale.it/eli/id/2015/02/18/15A01032/sg). 
quando lo Stato chiede di compilare un modulo che però non protegge la pri$v a c y$, e che espone a ulteriori rischi. Infatti, chi abita in un piccolo Comune può trovarsi in situazioni spiacevoli se, ad esempio, la persona che controlla il suo documento è un/a concittadino/a che ha una relazione di amicizia/parentela con il maltrattante e provvede a informarlo (Ruggiu e Console, 2020 b). Peraltro, la questione dell'autocertificazione e del distanziamento sociale colpisce la donna una seconda volta perché può ricevere una multa e perché la costringe ad affrontare il percorso da sola, senza amiche che possano accompagnarla (che cosa mai potrebbero scrivere sul loro documento?).

Proseguiamo: immaginiamo che tale donna voglia fare ricorso a tutti i nodi della rete che solitamente si attiva in questi casi (servizi sociali, associazioni, sportelli informativi, ospedali, tribunali, forze dell'ordine, ecc.). Il loro funzionamento è stato temporaneamente limitato dalle circostanze. Anche questo è un tassello di violenza istituzionale: quando lo Stato non riesce a stare accanto a chi sta vivendo circostanze che possono degenerare drammaticamente in fretta. La donna si trova a dover chiedere aiuto presso uffici che operano con orari ridotti, tipo una volta alla settimana, e con meno personale; talvolta la convivenza 24 ore su 24 con il marito/compagno, e il suo eventuale controllo, possono renderle impossibile anche solo il telefonare a servizi che abbiano orari limitati e prefissati, per non parlare del recarsi presso di loro. In questo caso lo Stato non tiene in dovuto conto che non è facile denunciare il maltrattante, nonostante tutte le ricerche che lo dimostrano, anche ripetute a distanza di anni (Istat, 2006 e 2014). Una serie di ragioni rende questa decisione sofferta, e la donna che giunge a prenderla, o che ha il coraggio di nominare la violenza ad altre persone per cercare assistenza, avrebbe il diritto di essere favorita in modo sollecito.

Mettiamo infine che, passando i giorni, questa donna si trovi in pericolo di vita e il Centro antiviolenza riesca a organizzare il suo trasferimento in una Casa di accoglienza ${ }^{7}$. Lei potrebbe essere positiva al Coronavirus senza saperlo, e potrebbe diffonderlo alle altre ospiti della Casa. Oppure, le altre donne, e $\mathrm{i}$ bambini già presenti, potrebbero essere positivi senza sintomi e la potrebbero contagiare. In questo caso si palesa un altro tassello di violenza istituzionale, che si esprime quando lo Stato espone la donna, e la sua eventuale prole, a un rischio sanitario.

Per riassumere, $i$ tre punti esemplificati hanno impensierito da subito $i$ Centri antiviolenza, le associazioni femminili e i vari attori sociali della rete di supporto: che cosa va indicato nell'autocertificazione quando una vittima va a

${ }^{7}$ L' $85,8 \%$ dei Centri può contare su una casa rifugio, che serve per le situazioni più gravi, nelle quali la donna e i suoi figli corrono gravi pericoli. La casa rifugio è più spesso gestita dai CAV ma con il supporto della rete antiviolenza e/o dei servizi territoriali di competenza (Istat, 2017). 
chiedere aiuto; come mantenere disponibile la rete che assiste le donne, nonostante le difficoltà causate dal Covid-19; come organizzare le case rifugio, e quali protezioni sanitarie adottare negli ambienti che ospitano queste piccole comunità. Le tre istanze sono state portate all'attenzione dell'opinione pubblica attraverso comunicazioni sui social e sulla stampa; il 16 marzo 2020, poi, il D.i.Re-Donne in Rete contro la violenza si è rivolto direttamente alla Ministra per le Pari Opportunità e la Famiglia, Elena Bonetti.

Dal punto di vista politico, la gestione della situazione, con le nuove consapevolezze, si è messa in moto verso la metà del mese di marzo. I dubbi principali sono stati chiariti proprio dalla Ministra alle Pari opportunità e dalla Ministra dell'Interno, Luciana Lamorgese.

Il 21 marzo, la Ministra Bonetti si è espressa sull'autocertificazione che chiunque deve mostrare, durante i controlli, per motivare i propri movimenti fuori casa, dicendo che: «Le donne hanno diritto di recarsi nei Centri antiviolenza senza essere multate e senza dover dichiarare altro motivo che lo stato di necessità» (Ruggiu e Console, 2020 a). Nessuna multa, dunque; inoltre, la dicitura di uno spostamento per "stato di necessità" non fa correre rischi se la documentazione viene trovata dal marito/compagno.

Quanto alla seconda istanza, a proposito delle case di accoglienza, Bonetti ha poi garantito fondi aggiuntivi ai Centri antiviolenza: per l'acquisto del materiale sanitario in grado di proteggere le ospiti e le operatrici, per la formazione specifica al personale e, soprattutto, per l'adeguamento delle strutture. Queste ultime devono prevedere stanze idonee ad alloggiare le donne di nuovo accesso, che potrebbero essere già positive al virus o che potrebbero venire contagiate dalle altre ospiti già presenti nelle strutture.

Sempre sulle case rifugio, e sempre il 21 marzo 2020, la Ministra dell'Interno Luciana Lamorgese ha inviato una Circolare a tutte le Prefetture per sollecitare l'individuazione, e la messa a disposizione dei Centri, di alloggi che garantiscano la protezione sanitaria (Ministero dell'interno, 2020, Circolare n. 15350/117-2). Tali alloggi possono essere reperiti coinvolgendo i Comuni, gli enti e le associazioni del territorio, oppure possono essere requisiti, e devono essere riservati a quelle donne che si trovano «in sorveglianza sanitaria e isolamento fiduciario e in permanenza domiciliare, quando queste misure non possano, come nel caso specifico, essere attuate nel domicilio della persona interessata».

Per testimoniare che non si tratta di sofismi, va menzionata la storia di "Marta", una donna del milanese che, nel mese di marzo 2020, è stata minacciata di morte e ha denunciato il marito. È riuscita a chiamare un Centro antiviolenza ed è scappata di casa. Sperava di trovare ospitalità in una struttura protetta ma non è stata accolta perché le è stato chiesto l'esito di un tampone, al fine di verificare l'assenza del virus Covid-19. Il medico però non lo ha 
eseguito, dal momento che la donna non presentava i sintomi, così come viene richiesto dalle norme (Lanzetti, 2020). "Marta" ha dovuto vivere in albergo fino a quando la notizia è comparsa sui giornali, poi la generosità delle lettrici e dei lettori, e l'interesse della Ministra Bonetti, hanno risolto la situazione.

Quest'ultima vicenda meriterebbe una più ampia trattazione, dal momento che prefigura il consueto schema: l'allontanamento delle donne dalla loro casa e non quello, invece, del marito/compagno violento. Sono poche le realtà italiane nelle quali accade il contrario. Tra esse, va segnalata la presa di posizione del Procuratore di Trento, Sandro Raimondi, che è intervenuto proprio durante l'epidemia di Covid-19. Ha infatti dichiarato che: «le situazioni di pericolo contingente siano risolte attraverso una collocazione dei maltrattanti presso un domicilio diverso da quello dove vivono i componenti del nucleo familiare che subiscono violenza domestica». In questo modo, si evita di far correre rischi sanitari a donne e bambini che dovrebbero trasferirsi nelle strutture protette (Nepitelli, 2020).

Rispetto infine alla rete territoriale, pur nella complicazione del periodo, si cerca comunque di assicurare il contatto con le donne. Così si esprime Angela Romanin, Presidente del Coordinamento dei Centri antiviolenza dell'EmiliaRomagna:

proseguono i colloqui con le donne già in percorso e con le ospiti nelle case rifugio, come pure tutti i contatti con la rete di supporto (servizi sociali, FFOO, Pronto soccorso, avvocate, ecc.) gestiti con nuove modalità dettate dalle misure di sicurezza, che prediligono il colloquio telefonico o la videochiamata, riservando i colloqui di persona alle sole emergenze (Bertossi, 2020).

La Ministra Bonetti, in risposta alla lettera sopracitata di Dire, oltre a garantire l'attribuzione dei fondi 2019 (non ancora pervenuti) e 2020, ha chiarito che: le case di accoglienza e i Centri restano aperti nonostante il Covid-19; il numero telefonico 1522 continua a funzionare 24 ore su 24; c'è una terza possibilità per chiedere sostegno, l'apposita $A P P 1522$ da scaricare nel telefono cellulare.

Per risolvere il calo delle denunce, invece, il 28 marzo 2020 la Ministra Lamorgese ha rilanciato la app YouPol, che in origine era stata pensata per i giovani, affinché denunciassero episodi di bullismo e spaccio di droga a scuola. La app, in effetti, garantisce un maggiore silenzio nell'utilizzo; le donne possono utilizzarla quando sono chiuse in bagno, ad esempio, e può trasmettere messaggi e immagini agli operatori (Polizia di Stato, 2020 b) ${ }^{8}$.

\footnotetext{
${ }^{8}$ Le segnalazioni possono essere fatte in anonimato. Sono georeferenziate, ma la donna può modificare il luogo dove sono avvenuti i fatti. La app può essere usata dalla vittima o da testimoni (tipo i vicini di casa).
} 
Tuttavia, resta una questione da risolvere: quando la violenza istituzionale si esprime con i mancati finanziamenti. I Centri, infatti, come detto sopra, attendono ancora dallo Stato i fondi stanziati per l'anno 2019, alla data del 30 marzo 2020 non sono ancora stati distribuiti. Inoltre, sullo sfondo si intravvedono già gli altri problemi che verranno: il prevedibile aumento di richieste di aiuto al termine dell'isolamento domiciliare, e la questione dell'indipendenza economica, indispensabile per le donne che tentano di uscire dalla violenza, a fronte di un mercato del lavoro che ripartirà a fatica. Mercato del lavoro in cui, in aggiunta, le donne sono un anello debole, occupando in maggior proporzione i posti con contratti atipici, dequalificati e a tempo determinato, insomma: posizioni precarie, che certo non aiutano le vittime nel progettarsi serenamente.

\section{Conclusioni pedagogiche}

Nei primi mesi dell'epidemia di Covid-19, la situazione nel nostro paese si è mostrata drammatica sotto molti punti di vista ma non ha silenziato l'esigenza collettiva di preoccuparsi anche della violenza domestica. I vertici dello Stato italiano durante tale periodo hanno praticato forme di violenza istituzionale ma, grazie alle sollecitazioni provenienti dai Centri antiviolenza, dalle associazioni di donne e dal discorso pubblico, hanno saputo rimediare modificando le norme e le prassi. In particolare, l'inversione di rotta è stata guidata da due Ministre della Repubblica.

Rispetto al passato, c'è oggi un'attenzione più vigile sul "problema violenza", che fa mobilitare le cittadine e i cittadini. Alcuni messaggi sono stati recepiti, soprattutto a proposito degli abusi intrafamiliari, tra i quali: non si esce da sole dalla situazione, e serve il concorso di molte forze sociali in campo. Talvolta alcune di queste forze possono "distrarsi", diciamo così, possono essere noncuranti o trascuranti, fino ad arrivare a praticare una violenza istituzionale. Ma durante l'epidemia si è visto che c'è stata una reazione sana da parte della popolazione, che si è fatta carico di attivarsi velocemente per chiedere il ripristino delle tutele.

Meno studiata rispetto alle altre tipologie di violenza di genere, quella istituzionale può sembrare distante, difficile da risolvere. Tuttavia, il processo di appropriazione del problema da parte del senso comune, che questa vicenda ha mostrato in azione, esorta a continuare a lavorare, nei contesti educativi, verso il cambiamento. Quando un tema così complesso diviene patrimonio collettivo (di molti, almeno) significa proprio che è avvenuta, o si sta realizzando, un'evoluzione culturale. Il contributo pedagogico è cruciale nel continuare tale progresso, ed esso deve essere impostato sullo studio, sulle ricerche 
e sulla formazione sia a proposito dei «cerchi concentrici» più vicini sia di quelli più lontani:

Ne deriva, per noi pedagogisti, un richiamo all'impegno di «lavorare» sia sul terreno politico/culturale - testimoniando la nostra «resistenza» nei confronti dei condizionamenti del «contesto che non c'è» - sia su quello più strettamente educativo, per affermare decisamente l'obiettivo etico del «realizza te stesso realizzando l'altro» e per tendere al superamento dell'analfabetismo emozionale nonché dell'afasia in tema di rapporti di convivenza e d'amore (Contini, 2009, p. 33).

Se già il lavoro educativo mira a implementare «l'etica dell'interconnessione» - che deve caratterizzare una comunità dove si realizza un rapporto di reciprocità tra donne e uomini, dove non c'è gerarchia tra i sessi (che poi è uno dei precursori della violenza) e dove non si verificano esclusioni sociali da parte di un gruppo rispetto all'altro (Pinto Minerva, 2013 b) si tratta di impostarlo spingendo lo sguardo anche in una direzione meno battuta. Se vogliamo, può essere considerata una direzione collaterale, appunto un affondo, un approfondimento, ma all'interno di un quadro di interesse già avviato. Tra tutti gli obiettivi che si pongono le analisi pedagogiche, quello relativo alla violenza istituzionale (il livello macro) potrebbe infatti rientrare in questo: «avviare una vasta azione di decostruzione, decondizionamento e di un vero e proprio disapprendimento delle logiche discorsive e delle pratiche di potere stratificate nella storia delle culture» (Pinto Minerva, 2013 b, p. 22). L'analisi delle «pratiche del potere», e dunque anche dell'intervento dello Stato, è già in nostro possesso, dal momento che i saperi pedagogici si pongono la finalità di:

presidiare - a tutti i livelli dello Stato, della magistratura, dei servizi della scuola e dell'università, della famiglia, dei rapporti tra le nazioni, le culture e le diverse religioni - che esso [il potere] non venga gestito con modalità violente e irrispettose. Il potere può essere anche dono: di esempio, sostegno, emancipazione, promozione, presa in carico, assunzione di responsabilità (Corsi e Riva, 2013 b, p. 6).

Nell'impostare i nuovi progetti educativi contro la violenza sulle donne, in grado di accompagnare le giovani generazioni verso una trasformazione di sensibilità e comportamenti, si terrà conto anche di ciò che l'epidemia ha fatto emergere, e la formazione potrà essere improntata pure sulla decostruzione del piano macro, finanche quando esso si esprime nelle forme istituzionali, che sembrano le più neutre possibili. 


\section{Bibliografia}

Bronfenbrenner U. (1979). The ecology of human development: experiments by nature and design. Cambridge, MA: Harvard University Press (trad. it: Ecologia dello sviluppo umano. Bologna: il Mulino, 2002).

Bronfenbrenner U. (Ed) (2005). Making human beings human: Bioecological perspectives on human development. Sage Publications Ltd (trad. it: Rendere umani gli esseri umani: bioecologia dello sviluppo. Trento: Erickson, 2010).

De Luca M. N. (2020). Marianna Manduca denunciò il marito ma fu uccisa. E ora lo Stato rivuole $i$ soldi dagli orfani. Testo disponibile al sito: https://rep.repubblica.it/pwa/generale/2020/02/10/news/marianna_denuncio_il_ma rito_ma_fu_uccisa_e_ora_lo_stato_rivuole_i_soldi_dagli_orfani- $248284438 / \overline{\text { [data }}$ di consultazione: $3 \overline{0}$ marzo 2020$]$.

Bertossi E. (2020). Coronavirus e violenza sulle donne: "Molte sono chiuse in casa con chi le maltratta». Testo disponibile al sito: http://www.bolognatoday.it/cronaca/coronavirus-violenza-donne-comedenunciare. $\mathrm{html}$ [data di consultazione: 30 marzo 2020].

Cambi F., Giosi M., Mariani A. e Sarsini D. (2009). Pedagogia generale. Identità, percorsi, funzione. Roma: Carocci.

Chiale S. (2020). Violenza sulle donne, cresce il sommerso: "Difficile chiedere aiuto». Testo disponibile al sito: https://milano.corriere.it/notizie/cronaca/20 marzo_31/violenza-donne-crescesommerso-difficile-chiedere-aiuto-8fd98d50-7340-11 ea-bc49338bb9c7b205.shtml [data di consultazione: 31 marzo 2020].

Ciccone S. (2009). Essere maschi. Tra potere e libertà. Torino: Rosemberg\&Sellier.

Contini M. (2002). La comunicazione intersoggettiva tra solitudini e globalizzazione. Firenze: La Nuova Italia.

Contini M. (2009). Le Famiglie oggi: problematicità e prospettive di cambiamento. Rivista Italiana di Educazione familiare, 1(2): 28-37. Doi: 10.13128/RIEF-3048.

Corsi M. e Riva M. G., a cura di (2013 a). Pedagogia Oggi. Violenze visibili e invisibili. Per una pedagogia dello svelamento e dell'impegno, 2.

Corsi M. e Riva M. G. (2013 b). La pedagogia della non violenza, oggi: riflessioni e proposte. In: EADS, a cura di, Pedagogia Oggi. Violenze visibili e invisibili. Per una pedagogia dello svelamento e dell'impegno, 2.

Corsi M., Fabbri F. e Riva M. G., a cura di (2016 a). Pedagogia oggi. Educare le emozioni. Contro la violenza, 1.

Corsi M., Fabbri F. e Riva M. G. (2016 b). Editoriale. In EADS, a cura di, Pedagogia oggi. Educare le emozioni. Contro la violenza, 1.

Cretella C. e Romero D. F. (2017). Parla con lei. Dibattiti sulla violenza contro le donne tra Italia e Spagna. Bologna: Edizioni Casa delle donne per non subire violenza.

Cretella C. e Sanchez I. M. (2014). Lessico familiare. Per un dizionario ragionato della violenza contro le donne. Pesaro: Settenove.

Cretella C. e Venturoli C., a cura di (2010). Voci dal verbo violare. Analisi e sfide educative contro la violenza sulle donne. Bologna: Odoya. 
Galelli R. (2014). Dispositivi biopolitici ed espropriazione dell'esperienza del corpo. In: Ulivieri S., a cura di, Corpi violati. Condizionamenti educativi e violenze di genere. Milano: FrancoAngeli.

Istat (2006). La violenza contro le donne dentro e fuori la famiglia - anno 2006. Testo disponibile al sito: https://www.istat.it/it/files//2011/07/testointegrale.pdf

[data di consultazione: 20 marzo 2020].

Istat (2014). La violenza contro le donne dentro e fuori la famiglia - anno 2014. Testo disponibile al sito: https://www.istat.it/it/files//2015/06/Violenze_contro_le_donne.pdf [data di consultazione: 20 marzo 2020].

Istat (2019). Rapporto I Centri Antiviolenza-Anno 2017. Testo disponibile al sito: https://www.osservatoriodiritti.it/wp-content/uploads/2019/11/violenza-sulledonne-1.pdf [data di consultazione: 20 marzo 2020].

Lanzetti E. (2020). Fugge dal compagno violento. «Senza test non trovo rifugio». Corriere della sera, 4 aprile.

Leonelli S. (2010), Per un'educazione al cambiamento contro la violenza. In: Cretella C. e Venturoli C., a cura di, Voci dal verbo violare. Analisi e sfide educative contro la violenza sulle donne, Bologna: I libri di Emil.

Leonelli S. (2014). La violenza nelle relazioni sentimentali degli adolescenti. In: Ulivieri S., a cura di, Corpi violati. Condizionamenti educativi e violenze di genere. Milano: FrancoAngeli.

Mind the gap (2020). Coronavirus, ministra Bonetti assicura: sempre attivo il numero 1522 contro la violenza sulle donne. Testo disponibile al sito: https://www.ilmessaggero.it/mind_the_gap/coronavirus_violenza_donne_feminici di_antiviolenza_1522_mind_the_gap-5118983.html [data di consultazione: 30 marzo 2020].

Morin E. (2001). L'Humanité de l'humanité : L'identité humaine. Paris: Le Seuil (trad. it: Il metodo 5. L'identità umana. Milano: Raffaello Cortina, 2002).

Musi E. (2014). Le radici nascoste della violenza. In: Ulivieri S., a cura di, Corpi violati. Condizionamenti educativi e violenze di genere. Milano: FrancoAngeli.

Nepitelli B. (2020). Violenza sulle donne: a Trento direttiva per allontanare $i$ maltrattanti. Testo disponibile al sito: https://alleyoop.ilsole24ore.com/2020/03/31/violenza-donne-trento/

[data di consultazione: 30 marzo 2020].

Offredi M. (2020). Violenza sulle donne, l'appello: «No al silenzio». I numeri da chiamare. Testo disponibile al sito: https://bergamo.corriere.it/notizie/cronaca/20_aprile_02/violenza-donne-1-appellono-silenzio-numeri-chiamare-ef8eb03a-751c-11 ea-b9 $\bar{c} 4-182209 \mathrm{~d} 6 \mathrm{cca} 4 . \operatorname{shtml}$ [data di consultazione: 2 aprile 2020].

Pinto Minerva F. (2013 a). La violenza sulle donne. In: Pedagogia Oggi. Violenze visibili e invisibili, Per una pedagogia dello svelamento e dell'impegno, 2.

Pinto Minerva F. (2013 b). Corpi feriti. La violenza sulle donne. In Cagnolati A., Pinto Minerva F. e Ulivieri S., a cura di, Le frontiere del corpo. Mutamenti e metamorfosi, Pisa: ETS. 
Polizia di Stato (2020 a). Rapporto Questo non è amore 2019. Testo disponibile al sito: https://www.poliziadistato.it/statics/12/brochure_questononeamore_2019.pdf [data di consultazione: 29 marzo 2020].

Polizia di Stato (2020 b). Youpol: sull'app della Polizia si possono segnalare anche le violenze domestiche. Testo disponibile al sito: https://www.poliziadistato.it/articolo/135e74a0112e9af858848025 [data di consultazione: 29 marzo 2020].

Quaranta I. (2006). Antropologia medica. I testi fondamentali. Milano: Raffaello Cortina.

Romito P. (1999). Dalla padella alla brace. Donne maltrattate, violenza privata e complicità pubbliche. Polis, 2. DOI: 10.1424/2715.

Romito P., a cura di (2000). Violenze alle donne e risposte delle istituzioni. Prospettive internazionali. Milano: FrancoAngeli.

Ruggiu V. e Console S. (2020, a). Coronavirus, la ministra Bonetti alle donne: "Se subite violenza, chiedete aiuto. Non temete l'autocertificazione». Testo disponibile al sito: https://www.repubblica.it/cronaca/2020/03/21/news/elena bonetti_se_subite_viole nza_chiedete_aiuto_andate_al_centro_antiviolenza_e_nessuno_vi_multera_$251867069 /$ [data di consultazione: 30 marzo 2020].

Ruggiu V. e Console S. (2020, b). Coronavirus, i Centri antiviolenza: «Le donne non riescono a chiederci aiuto. E noi siamo senza fondi». Testo disponibile al sito: https://www.repubblica.it/cronaca/2020/03/21/news/coronavirus_crollano_le_chia ma-

te_ai_centri_antiviolenza_le_donne_non_riescono_a_chiedere_aiuto_e_noi_siamo

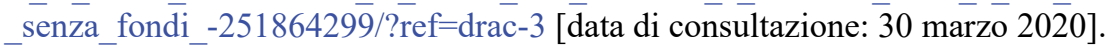

Onu (1993). Dichiarazione sull'eliminazione della violenza contro le donne. Testo disponibile al

sito: https://www.esteri.it/mae/approfondimenti/20090827 allegato2 it.pdf [data di consultazione: 30 marzo 2020].

Onu (2020). States must combat domestic violence in the context of COVID-19 lockdowns - UN rights expert - 27 March. Testo disponibile al sito: https://www.ohchr.org/EN/NewsEvents/Pages/DisplayNews.aspx?NewsID=25749 $\&$ LangID $=\mathrm{E}$ [data di consultazione: 30 marzo 2020].

Spinelli B. (2008). Femminicidio. Dalla denuncia sociale al riconoscimento giuridico internazionale. Milano: FrancoAngeli.

Ulivieri S. (2013 a). Il corpo delle donne e la violenza di genere. Il segno di uno storico dominio. In: Cagnolati A., Pinto Minerva F. e Ulivieri S., a cura di, Le frontiere del corpo. Mutamenti e metamorfosi, Pisa: ETS.

Ulivieri S. (2013 b). Femminicidio e violenza di genere. In: Pedagogia Oggi. Violenze visibili e invisibili, Per una pedagogia dello svelamento e dell'impegno, 2.

Ulivieri S., a cura di (2014). Corpi violati. Condizionamenti educativi e violenze di genere. Milano: FrancoAngeli. 


\section{Provvedimenti legislativi sull'emergenza Covid-19 pertinenti al tema $\mathrm{e}$ in ordine cronologico (fino al 30 marzo 2020):}

Ordinanza del Ministro della salute 21 febbraio 2020, pubblicata nella Gazzetta Ufficiale della Repubblica italiana - Serie generale - n. 44 del 22 febbraio 2020. Testo disponibile al sito: http://www.salute.gov.it/imgs/C_17_notizie_4283_0_file.pdf [data di consultazione: 30 marzo 2020].

Decreto-legge 23 febbraio 2020, n. 6, recante «Misure urgenti in materia di contenimento e gestione dell'emergenza epidemiologica da COVID-19». Testo disponibile al sito: https://www.gazzettaufficiale.it/eli/id/2020/02/23/20G00020/sg [data di consultazione: 30 marzo 2020].

Decreto del Presidente del Consiglio dei ministri 23 febbraio 2020, recante «Disposizioni attuative del decreto-legge 23 febbraio 2020, n. 6, recante misure urgenti in materia di contenimento e gestione dell'emergenza epidemiologica da COVID19», pubblicato nella Gazzetta Ufficiale n. 45 del 23 febbraio 2020. Testo disponibile al sito: https:/www.gazzettaufficiale.it/eli/id/2020/02/23/20A01228/sg [data di consultazione: 30 marzo 2020].

Decreto del Presidente del Consiglio dei ministri 25 febbraio 2020, recante «Ulteriori disposizioni attuative del decreto-legge 23 febbraio 2020, n. 6, recante misure urgenti in materia di contenimento e gestione dell'emergenza epidemiologica da COVID-19», pubblicato nella Gazzetta Ufficiale n. 47 del 25 febbraio 2020. Testo disponibile al sito: https://www.gazzettaufficiale.it/eli/id/2020/02/25/20A01278/sg [data di consultazione: 30 marzo 2020].

Decreto del Presidente del Consiglio dei ministri $1^{\circ}$ marzo 2020, recante «Ulteriori disposizioni attuative del decreto-legge 23 febbraio 2020, n. 6, recante misure urgenti in materia di contenimento e gestione dell'emergenza epidemiologica da COVID-19», pubblicato nella Gazzetta Ufficiale n. 52 del $1^{\circ}$ marzo 2020. Testo disponibile al sito: https://www.gazzettaufficiale.it/eli/id/2020/03/01/20A01381/sg [data di consultazione: 30 marzo 2020].

Decreto del Presidente del Consiglio dei ministri 4 marzo 2020, recante «Ulteriori disposizioni attuative del decreto-legge 23 febbraio 2020, n. 6, recante misure urgenti in materia di contenimento e gestione dell'emergenza epidemiologica da COVID-19, applicabili sull'intero territorio nazionale», pubblicato nella Gazzetta Ufficiale n. 55 del 4 marzo 2020. Testo disponibile al sito: https://www.gazzettaufficiale.it/eli/id/2020/03/04/20A01475/sg [data di consultazione: 30 marzo 2020].

Decreto del Presidente del Consiglio dei ministri 8 marzo 2020, recante «Ulteriori disposizioni attuative del decreto-legge 23 febbraio 2020, n. 6, recante misure urgenti in materia di contenimento e gestione dell'emergenza epidemiologica da COVID-19, applicabili sull'intero territorio nazionale», pubblicato nella Gazzetta Ufficiale n. 59 dell'8 marzo 2020. Testo disponibile al sito: https://www.gazzettaufficiale.it/eli/id/2020/03/08/20A01522/sg [data di consultazione: 30 marzo 2020].

Decreto del Presidente del Consiglio dei ministri 9 marzo 2020, recante «Ulteriori disposizioni attuative del decreto-legge 23 febbraio 2020, n. 6, recante misure ur- 
genti in materia di contenimento e gestione dell'emergenza epidemiologica da COVID-19», pubblicato nella Gazzetta Ufficiale n. 62 del 9 marzo 2020”. Testo disponibile al sito: https://www.gazzettaufficiale.it/atto/vediMenuHTML?atto.dataPubblicazioneGazz etta $=2020-03-$

09\&atto.codice Redazionale $=20$ A $01558 \&$ tipoSerie $=$ serie_generale $\&$ tipoVigenza $=$ originario [data di consultazione: 30 marzo 2020].

Decreto del Presidente del Consiglio dei ministri 11 marzo 2020, recante «Ulteriori disposizioni attuative del decreto-legge 23 febbraio 2020, n. 6, recante misure urgenti in materia di contenimento e gestione dell'emergenza epidemiologica da COVID-19, applicabili sull'intero territorio nazionale», pubblicato nella Gazzetta Ufficiale n. 64 del 11 marzo 2020. Testo disponibile al sito: https://www.gazzettaufficiale.it/eli/id/2020/03/11/20A01605/sg [data di consultazione: 30 marzo 2020].

Ordinanza del Ministro della salute del 20 marzo 2020 recante «Ulteriori misure urgenti in materia di contenimento e gestione dell'emergenza epidemiologica da COVID-19, applicabili sull'intero territorio nazionale» pubblicata nella Gazzetta Ufficiale n. 73 del 20 marzo 2020. Testo disponibile al sito: https://www.gazzettaufficiale.it/eli/id/2020/03/20/20A01797/sg [data di consultazione: 30 marzo 2020].

Ministero dell'interno. Circolare n. 15350/117(2) del 21 marzo 2020. Polmonite da nuovo coronavirus (COVID - 19). Accoglienza donne vittime di violenza. Testo disponibile al

sito: https://www.interno.gov.it/sites/default/files/covid circolare vittime_violenza.pdf [data di consultazione: 27 marzo 2020].

Ordinanza del Ministro dell'interno e del Ministro della salute del 22 marzo 2020 recante «Ulteriori misure urgenti in materia di contenimento e gestione dell'emergenza epidemiologica da COVID-19, applicabili sull'intero territorio nazionale». Testo disponibile al sito: https://www.gazzettaufficiale.it/eli/id/2020/03/22/20A01806/sg [data di consultazione: 30 marzo 2020].

Decreto del Presidente del Consiglio dei ministri 22 marzo 2020, recante «Ulteriori disposizioni attuative del decreto-legge 23 febbraio 2020, n. 6, recante misure urgenti in materia di contenimento e gestione dell'emergenza epidemiologica da COVID-19, applicabili sull'intero territorio nazionale», pubblicato nella Gazzetta Ufficiale n. 76 del 22 marzo 2020. Testo disponibile al sito: https://www.gazzettaufficiale.it/eli/id/2020/03/22/20A01807/sg [data di consultazione: 30 marzo 2020] 